\title{
Management of Zygomatico-Orbital Fractures
}

\author{
Original Amr Amin Ghanem \\ Article \\ Lecturer of Oral and Maxillofacial Surgery, Faculty of Dentistry, Ain-Shams University, \\ Cairo, Egypt
}

\begin{abstract}
Twelve patients with unilateral ZMO fractures operated within 5-10 days of trauma according to Ellis and Perez were operated. Clinical and CT scan findings were used to evaluate the accuracy of the reduction as well as the esthetic outcome secondary to the surgical approaches used. The patients were photographed according to a standardized protocol pre operatively and 3 months postoperatively. A blinded panel of reviewers was formed for assessment of the surgical results. The panel was composed of two groups. A specialized group which encompassed three oral and maxillofacial surgery consultants as well as two oral and maxillofacial radiology consultants. A second group encompassed three dental school students who volunteered to participate in the study. There were direct correlation between percent of satisfaction for surgeon, Radiologists and non-experts. Moreover, there were direct correlation between percent of satisfaction for Radiologists and non-experts using the Pearson correlation coefficient. Advocation of the Ellis and perez algorithm yields satisfactory esthetic outcomes.
\end{abstract}

Key Words: Eye lid incisions, trauma, zygomatico orbital fractures

Received:17 April 2018, Accepted:29 July 2018

Corresponding Author: Amr Amin Ghanem, Lecturer of Oral and Maxillofacial Surgery, Faculty of Dentistry, Ain-Shams University, Cairo, Egypt, Tel.: 01112555297, E-mail: Amrghanem@asfd.asu.edu.eg

ISSN: 2090-097X, May 2018, Vol. 9, No. 2

\section{INTRODUCTION}

The anterolateral part of the facial skeleton is occupied by the zygomatic bone and its complex articulations. Trauma inflicted via motor vehicle accidents, assaults as well as falls or sports related injuries result in variable fracture patterns affecting this critical region of the face ${ }^{[1]}$.

Fractures of this region have been coined with different terms. The term Zygomatico-maxillary-orbital fractures (ZMO fix.) have been considered a more accurate term by different authorities. This is because the term isolates the simple isolated zygomatic arch fractures from the more complicated fractures of the zygomatic apparatus and its consequences on the orbital anatomy ${ }^{[2]}$.

The treatment of these fractures entails restoration of form as well as function. Access to these fractures is achieved via a group of facial surgical approaches. Selection of the appropriate surgical approach varies according to the pattern of the fracture ${ }^{[1-3]}$.

The surgical approaches utilized in the treatment of these fracture can be classified according to the anatomical target area. Approaches to the infraorbital rim and orbital floor entail: Subciliary, Sub-tarsal and Trans conjunctival approaches. Approaches to the lateral orbital wall include the Blepharoplastic upper eye lid incision as well as the lateral eye brow incision. The coronal axis can expose the lateral orbital wall, orbital roof as well as the medial orbital wall. The medial orbital wall can be approached via a retrocaruncular incision combined with a trans conjunctival approach. Furthermore, the Lynch incision as well as open sky incisions can be advocated for approaching the medial orbital wall. Existing lacerations can be utilized as a surgical access ${ }^{[4,2,1,3,5,6]}$.

A change in surgical concepts of ZMO fx. treatment started stimulated by the emergence of intraoperative imaging $^{[7,8]}$. Ellis and Perez in 2014 ${ }^{[2]}$ presented an algorithm for the management of ZMO fx. treatment. The algorithm entailed two axes. One could be applied if intraoperative imaging was available. The second axis was designed for operating rooms without the facility of intraoperative imaging. This protocol served to change the concepts behind selecting the surgical approaches to be utilized in open reduction and internal fixation. The algorithm suggests utilizing the approach that provides the best esthetic results while giving enough access for proper fixation. This concept has integrated the esthetic aspect into surgical decision making. The esthetic demand in management of $\mathrm{ZMO}$ fractures must be taken into consideration as it is one of the prime concerns of patients. The surgical concepts for long advocated wide exposure of ZMO fx. as well as routine three or four-point fixation of these fractures. The current practice includes customizing the surgical approaches and fixation points according to the fracture presentation. This change in concept can minimize 
many of the complications secondary to the surgical approaches utilized ${ }^{[9,8,4,1]}$.

\section{MATERIALS AND METHODS}

A retrospective analysis of twelve patients with unilateral ZMO fractures operated within 5-10 days of trauma according to Ellis and Perez were operated. Clinical and CT scan findings were used to evaluate the accuracy of the reduction as well as the esthetic outcome secondary to the surgical approaches used. The patients were photographed according to a standardized protocol pre operatively and 3 months postoperatively. Pre and postoperative CT scans over the facial bones were collected for all cases.
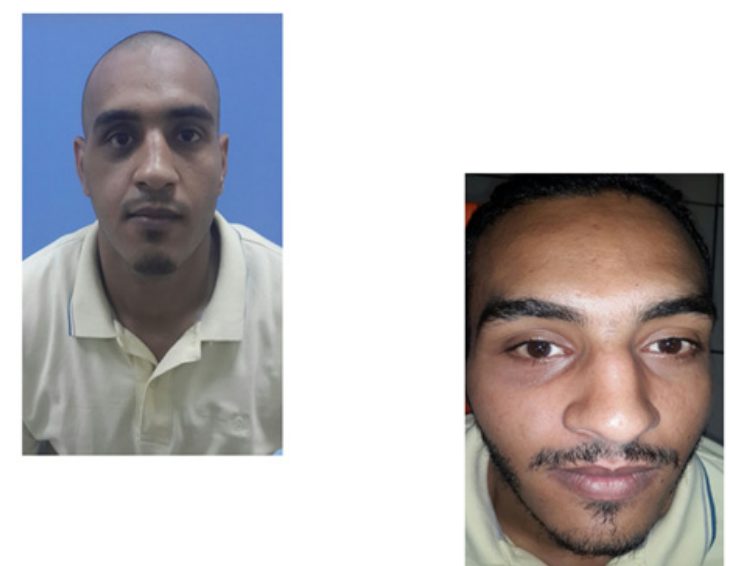

Fig. 1: Top left corner shows the preoperative view following Trauma, the bottom right corner shows the post-operative view 10 days post-surgery-A subtarsal incision was utilized.
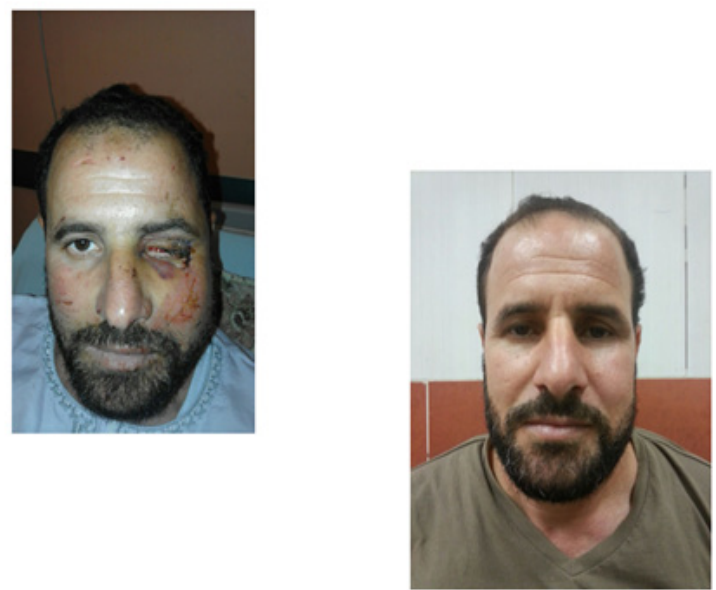

Fig. 2: Top left corner shows the preoperative view, bottom left corner shows the postoperative view- Subtarsal incision to expose the orbital floor
A blinded panel of reviewers was formed for assessment of the surgical results .The panel was composed of two groups. A specialized group which encompassed three oral and maxillofacial surgery consultants as well as two oral and maxillofacial radiology consultants. A second group encompassed three dental school students who volunteered to participate in the study.
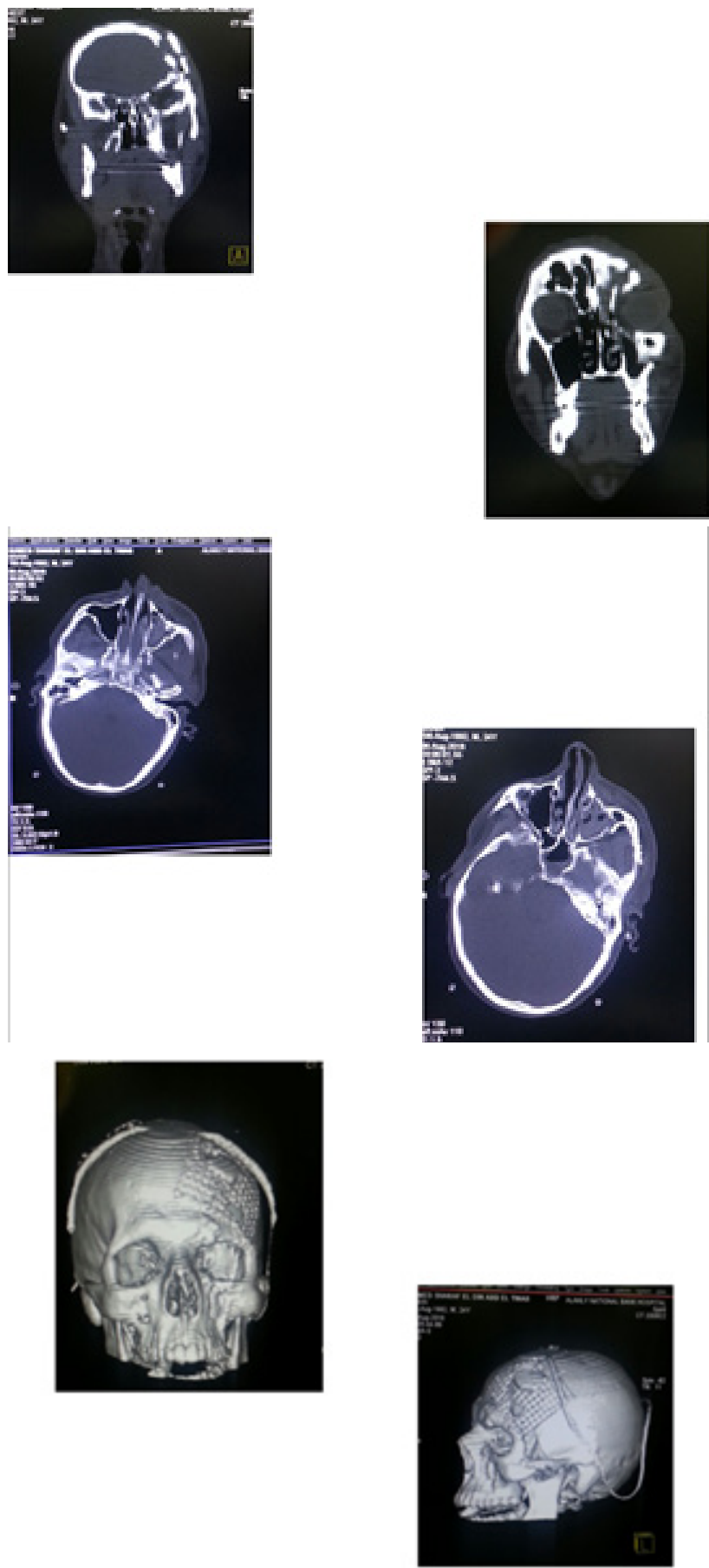

Fig. 3: Showing radiographic images as displayed in the power point presentation showed to the panel. 
The data for assessment was presented for all groups on individual basis. A power point presentation comprising the images as well as a questionnaire was provided to the panel member.

The clinical parameters included questions covering all aspects of the surgical procedure. Furthermore, the questionnaire also included questions concerning the radiographic data provided. All answers were in a form of grading system.

Data derived from clinical and radiographic assessments was be collected and tabulated. The grades provided by the panel were then subjected to statistical analysis. This assessment technique has been applied in similar studies $^{[5]}$.

\section{Questionnaire form}

Name:

Specialty:

\section{Please grade according to your opinion:}

- Healing of the surgical access site.

\begin{tabular}{|l|l|l|l|l|l|l|l|l|l|}
\hline & & & & & & & & & \\
\hline $\begin{array}{l}\text { Least } \\
\text { Satisfatory }\end{array}$ & 2 & 3 & 4 & 5 & 6 & 7 & 8 & 9 & $\begin{array}{l}\text { Most } \\
\text { Satisfatory }\end{array}$ \\
\hline
\end{tabular}

- Lower eye lid abnormalities

\begin{tabular}{|l|l|l|l|l|l|l|l|l|l|}
\hline & & & & & & & & & \\
\hline $\begin{array}{l}1 \\
\text { Satisfatory }\end{array}$ & 2 & 3 & 4 & 5 & 6 & 7 & 8 & 9 & $\begin{array}{l}10 \\
\text { Most } \\
\text { Satisfatory }\end{array}$ \\
\hline
\end{tabular}

- Facial esthetics in comparison with the un-operated side.

\begin{tabular}{|l|l|l|l|l|l|l|l|l|l|}
\hline & & & & & & & & & \\
\hline $\begin{array}{l}\text { Least } \\
\text { Satisfatory }\end{array}$ & 2 & 3 & 4 & 5 & 6 & 7 & 8 & 9 & $\begin{array}{l}\text { Most } \\
\text { Satisfatory }\end{array}$ \\
\hline
\end{tabular}

- Complications associated with applied surgical approach

\begin{tabular}{|l|l|l|l|l|l|l|l|l|l|}
\hline & & & & & & & & & \\
\hline $\begin{array}{l}\text { Least } \\
\text { Satisfatory }\end{array}$ & 2 & 3 & 4 & 5 & 6 & 7 & 8 & 9 & $\begin{array}{l}10 \\
\text { Most } \\
\text { Satisfatory }\end{array}$ \\
\hline
\end{tabular}

- Accuracy of the reduction. 


\begin{tabular}{|l|l|l|l|l|l|l|l|l|}
\hline & & & & & & & & \\
\hline $\begin{array}{l}\text { Least } \\
\text { Satisfatory }\end{array}$ & 2 & 3 & 4 & 5 & 6 & 7 & 8 & 9 \\
\hline
\end{tabular}

- Position of the Hard ware.

\begin{tabular}{|l|l|l|l|l|l|l|l|l|}
\hline & & & & & & & & \\
\hline $\begin{array}{l}\text { Least } \\
\text { Satisfatory }\end{array}$ & 2 & 3 & 4 & 5 & 6 & 7 & 8 & 9 \\
Matisfatory \\
\hline
\end{tabular}

- Accuracy of orbital floor reconstruction.

\begin{tabular}{|l|l|l|l|l|l|l|l|l|l|}
\hline & & & & & & & & & \\
\hline $\begin{array}{l}\text { Least } \\
\text { Satisfatory }\end{array}$ & 2 & 3 & 4 & 5 & 6 & 7 & 8 & 9 \\
\hline
\end{tabular}

- Grade the post-operative outcome.

\begin{tabular}{|l|l|l|l|l|l|l|l|l|l|}
\hline & & & & & & & & & \\
\hline $\begin{array}{l}\text { Least } \\
\text { Satisfatory }\end{array}$ & 2 & 3 & 4 & 5 & 6 & 7 & 8 & 9 \\
\hline
\end{tabular}

\section{RESULTS}

\section{Data Management and Analysis:}

The collected data was revised, coded, tabulated and introduced to a PC using Statistical package for Social Science (SPSS 15.0 for windows; SPSS Inc, Chicago, IL, 2001). Data was presented and suitable analysis was done according to the type of data obtained for each parameter.

\section{i. Descriptive statistics:}

Median.

Minimum and maximum values (range) for numerical data.

Mean \pm SD

Frequency and percentage of non-numerical data.

\section{ii. Analytical statistics:}

1. Chi-Square test was used to examine the relationship between two qualitative variables.
2. Fisher's exact Chi-Square test is computed when a table that does not result from missing rows or columns in a larger table has a cell with an expected frequency of less than 5.

3. Pearson Correlations was used to assess the strength of association between two quantitative variables. The correlation coefficient denoted symbolically " $r$ " defines the strength and direction of the linear relationship between two variables.

4. One-Way ANOVA test was used to assess the statistical significance of the difference between more than two study group means.

\section{- P-value: level of significance}

$-P>0.05$ : Non significant (NS).

$-P \leq 0.05$ : Significant (S).

- $P \leq 0.01$ : Highly significant (HS).

There were direct correlation between percent of satisfaction for surgeon, Radiologists and non-experts. 
Moreover, there were direct correlation between percent of satisfaction for Radiologists and non-experts using the Pearson correlation coefficient.

There were no statistically significant differences between satisfaction of the surgical outcome for surgeon,
Radiologists and non experts using fisher exact chi-square test.

There was no statistical significant difference between the grading of the surgical outcome given by the assessing panel using one way ANOVA .

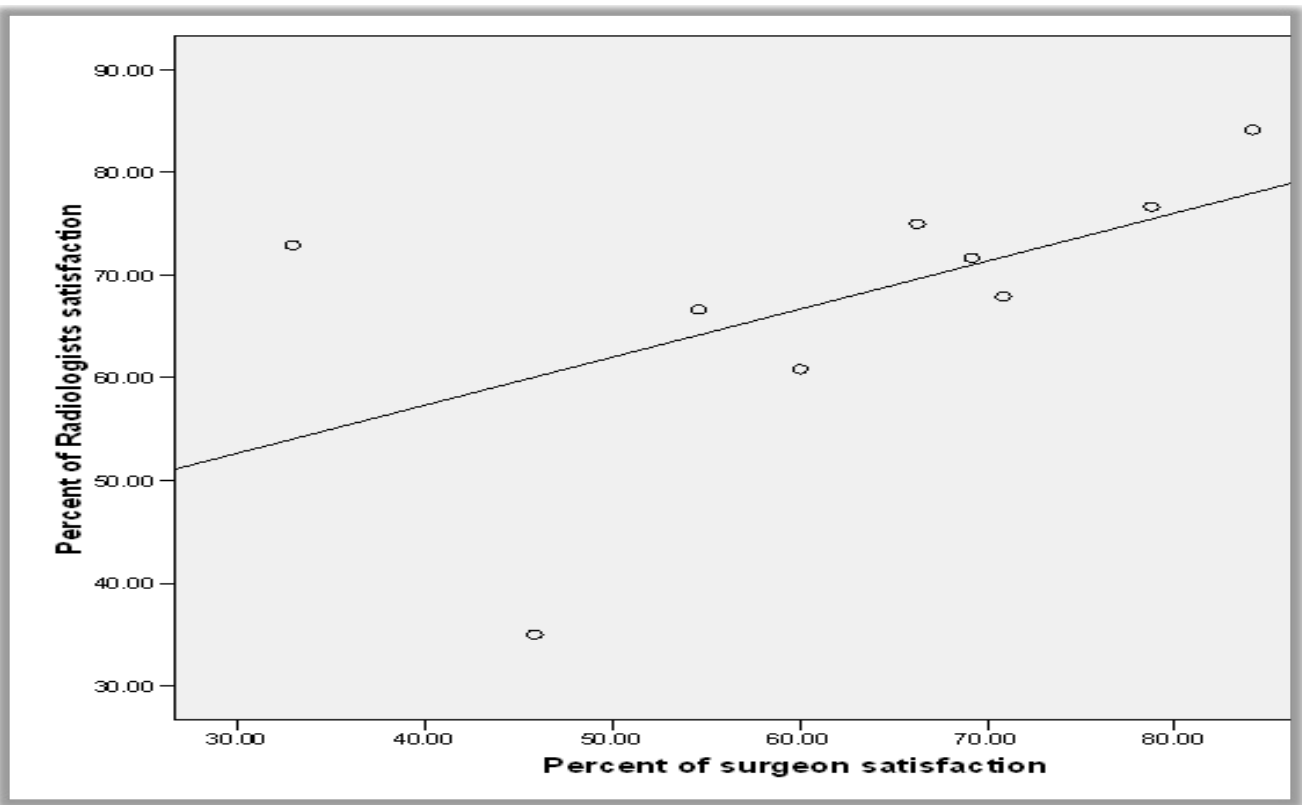

Fig. 4: Correlations between percent of satisfaction for surgeons and radiologist

Table 1: Correlations between percent of satisfaction for surgeons, radiologists and non experts

\begin{tabular}{llll}
\hline Percent of satisfaction & & Surgeon & Radiologists \\
& & & 1.0 \\
\hline Radiologists & $\mathrm{r}$ & 0.803 & - \\
& P value & 0.009 & - \\
& Sig. & HS & 0.895 \\
Non experts & $\mathrm{r}$ & 0.819 & 0.001 \\
& P value & 0.007 & HS \\
\hline
\end{tabular}

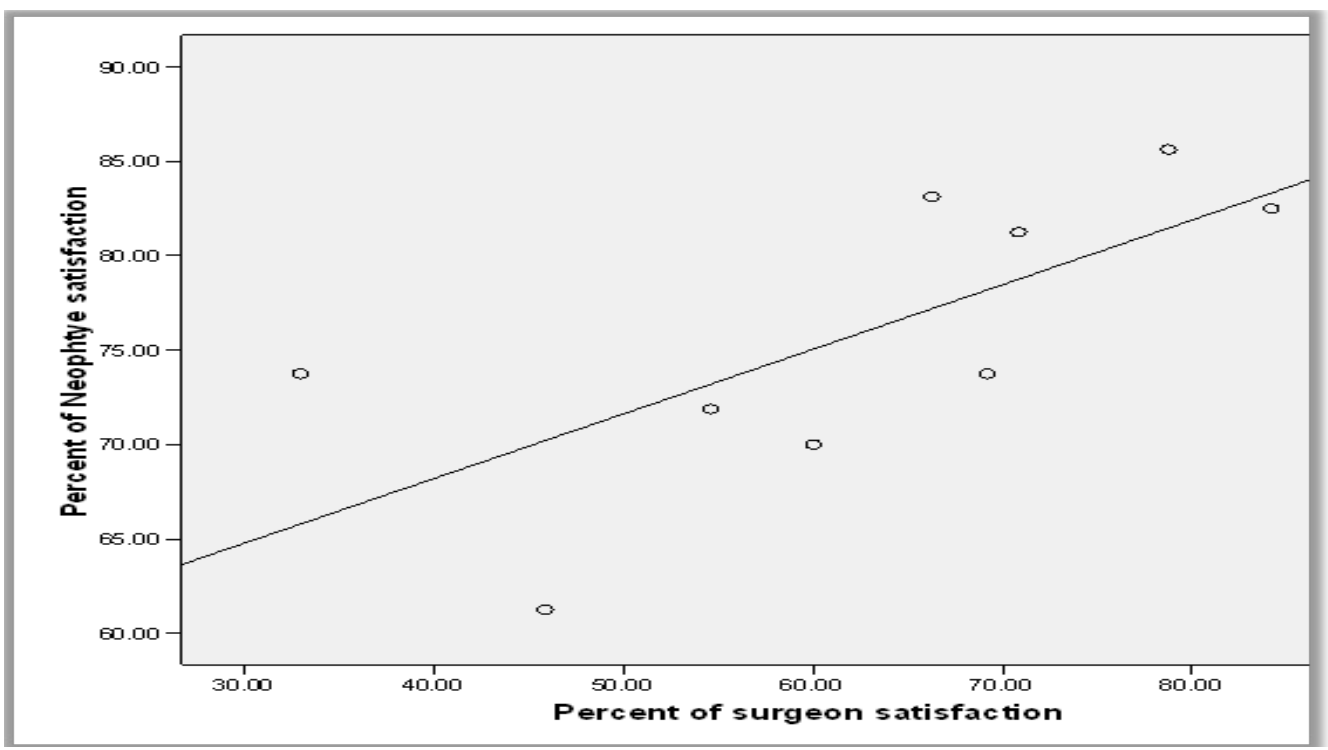

Fig. 5: Correlations between percent of satisfaction for surgeons and non-experts 
Table 2: Comparison between surgeons, radiologists and non experts regards satisfaction

\begin{tabular}{|c|c|c|c|c|c|c|c|}
\hline Accepted scale & $\begin{array}{l}\text { Surgeon } \\
(\mathrm{n}=9)\end{array}$ & $\begin{array}{l}\text { Radiologist } \\
(\mathrm{n}=9)\end{array}$ & $\begin{array}{l}\text { Non experts } \\
(\mathrm{n}=9)\end{array}$ & $\begin{array}{l}\text { Total } \\
(\mathrm{n}=27)\end{array}$ & $\mathrm{X}^{2}$ & P Value & Sig. \\
\hline Unacceptable & $1(11.1 \%)$ & $1(11.1 \%)$ & 0 & $2(7.4 \%)$ & 1.32 & 1.0 & NS \\
\hline Acceptable & $8(88.9 \%)$ & $8(88.9 \%)$ & $9(100 \%)$ & $25(92.6 \%)$ & & & \\
\hline
\end{tabular}

Fisher's exact Chi-Square test

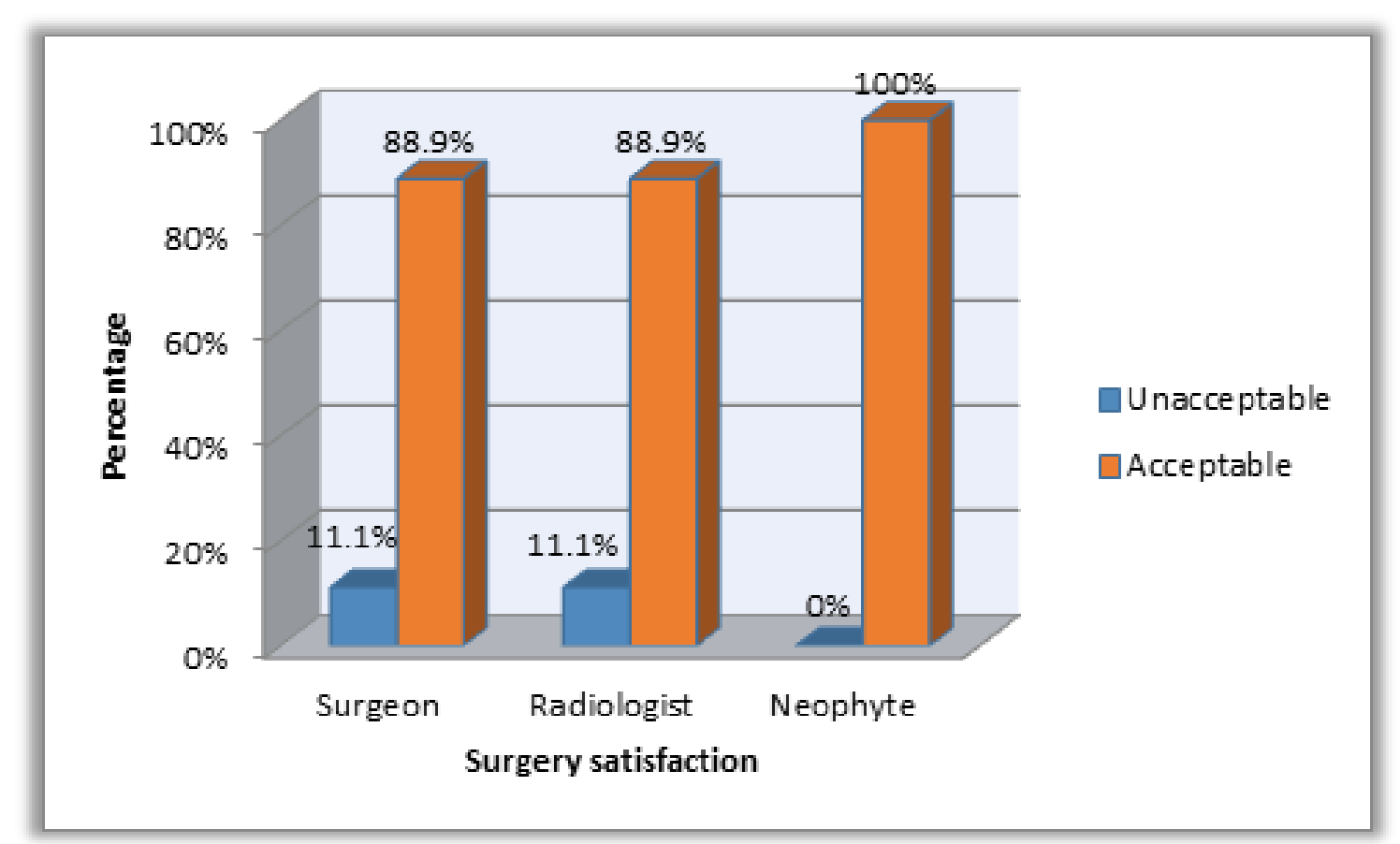

Fig. 6 :Bar chart representing comparison between surgeons, radiologists and non-experts regarding satisfaction percentages according to grades allocated by the panel.

Table 3: Comparison between percent of satisfaction for surgeons, radiologists and non-experts

\begin{tabular}{lccccccc}
\hline $\begin{array}{l}\text { percent of } \\
\text { satisfaction }\end{array}$ & Mean\% & SD & Median & Range & F & P Value & Sig. \\
\hline Surgeon & $63 \%$ & 16 & 66 & $33-84$ & & & \\
Radiologist & $68 \%$ & 14 & 72 & $35-84$ & 2.37 & 0.115 & NS \\
Non expert & $76 \%$ & 8 & 74 & $61-86$ & & \\
\hline
\end{tabular}

One-Way ANOVA 


\section{DISCUSSION}

ZMO fractures are associated with both an esthetic as well as a functional compromise. The selected treatment plan must address both aspects ${ }^{[2]}$. The esthetic aspect presents comprises two important sub topics. The accuracy of the fracture reduction. Moreover, the choice of the surgical access affects the final esthetic outcome ${ }^{[8,10]}$.

Regarding the reduction of the fractures, various reports offered different techniques of fracture reduction. The advocation of zygomatic hooks, Carroll-Girard screws as well as the use of a zygomatic reduction forceps had been reported. The effect of accurate reduction on the esthetic outcome has been studied thoroughly ${ }^{[4]}$.

A Poorly reduced ZMO fracture will impart the following facial features, depressed malar eminence, facial asymmetry, downward slanting of the eyelids on the affected side, the presence of bony contour anomalies as well as increased orbital volume. If there is an increase in the orbital volume, functional problems will emerge in addition to the previously mentioned esthetic complications $^{[1,11]}$.

The concern for esthetics also entails choosing the incisions that are likely to yield the most pleasant outcomes. The intraoral incision is one that leaves no conspicuous scars or postoperative complications. The incisions performed through the eye lids on the other hand are associated with risks worthy of study and consideration $^{[2]}$.

The subciliary, subtarsal and trans-conjunctival incision are namely the most advocate incisions. The subciliary incision is associated with denervation of the muscle fibers inserting on the tarsal plate. This has resulted in various degrees of lid shortening. the clinical presentation varies from increased scleral show to ectropion ${ }^{[3,5]}$.

Ridgway et al. reported an ectropion rate of $14 \%$ with subciliary incisions. The use of the subtarsal incision has been supported by various authors. The incision was first presented by John Converse in 1950. Distinguishing the ideal position of the subtarsal incision has been attempted by Feldman et al. this study concluded that in adults the incision can be easily placed in a skin crease while in younger patients $6-7 \mathrm{~mm}$ from the inferior tarsal plate was sufficient distance ${ }^{[6,10]}$.

The advocation of the transconjunctival incision is an esthetically appealing concept. However, as the need for increased exposure arises the use of a lateral canthotomy becomes a must. The lateral canthotomy is associated with later difficulties in positioning of the lateral canthal ligament. Spencer et al. presented a detailed discussion of these difficulties. The study concluded that most maxillofacial surgeons would be more comfortable closing a skin incision that re-suspending the lateral canthal ligament ${ }^{[2,5]}$.

A ZMO fracture may also be approached through a coronal flap. In the advent of lateral orbital wall comminuted fracture the coronal approach becomes necessary. This approach is associated with some complications. These include weakness of the temporal branch of the facial nerve as well as bleeding. Furthermore, alopecia sites can occur iatrogenic with these incisions ${ }^{[1]}$.

Lateral eye brow incisions and upper eye lid blepharoplastic incision may also be utilized in ZMO cases. These approaches are seldom associated with complications $^{[9,2,1]}$.

The functional aspect of these fractures is mainly associated with the orbit. Fractures in this distribution are associated with orbital manifestations including: diplopia, decreased visual acuity, ecchymosis, subconjunctival hemorrhage. In some cases, the occlusion may be disturbed secondary to a ZMO fracture ${ }^{[8,12]}$.

The current study aimed to assess an algorithm designed to minimize the use of extraoral incisions in ZMO management while reaching satisfactory surgical outcome. The outcome analysis was performed on three different levels. The level of experienced surgeons, maxillofacial radiologists as well as non-specialized observers.

The panel of experiences surgeons served to provide an assessment of the surgical decision and execution. The introduction of the sub tarsal incision and trans conjunctival retroseptal incision into practice was contrary to the regular use of subciliary incisions advocated by the assessing panel. The panel was blinded to the surgical approach used so as to prevent any experience bias.

The group of experienced radiologists was included in order to assess the accuracy of the reduction as well as the suitability of the fixation devices included. Finally, a group of non-specialized observers was included to concentrate on the esthetic outcome of surgery. This is because the esthetic outcome is also paramount to the patient undergoing treatment.

The panel performing the assessment was all blinded to the surgical team as well as the surgical decisions. The questions posed to the panel were all aiming at grading and quantifying the outcome. All groups showed high 
agreement with the surgical outcome. The difference between the groups was non-significant.

The non-specialized group should the highest acceptance which is regarded a normal finding. It is important to emphasize that this finding supports the return of the patients to normal function in the society.

The use of Ellis and Perez algorithm ${ }^{[2]}$ produced a satisfactory outcome as judged by the assessing panels. This supports a wider application of the protocol in order to minimize the advocation of extraoral approaches while maintaining acceptable surgical outcomes.

\section{CONFLICT OF INTEREST}

There are no conflicts of interest

\section{REFERENCES}

1. Current Management of Zygomaticomaxillary Complex Fractures:A Multidisciplinary Survey and Literature Review. Farber SJ, Nguyen DC, Skolnick GB, Woo AS, Patel KB. 4, Nov 2016, Craniomaxillofac Trauma Reconstr., Vol. 9, pp. 313-322.

2. An Algorithm for the Treatment of Isolated Zygomatico-Orbital Fractures. Ellis E, Perez D. 2014, J Oral Maxillofac Surg, Vols. 72:,, pp. 1975-1983.

3. Subciliary versus subtarsal approaches to orbitozygomatic fractures. Rohrich RJ, Janis JE, Adams WP Jr. 5, Apr 2003, Plast Reconstr Surg. , Vol. 111, pp. 1708-1714.

4. Fonseca, R. J. zygomatico Orbital Fractures. [book auth.] Ellis E 3rd. Oral and maxillofacial trauma. St. Louis, MO : Elsevier Saunders, (2005).

5. Subtarsal Versus Transconjunctival ApproachEsthetic and Functional Long-Term Experience. Strobel L, Hölzle F, Riediger D, Hilgers RD, Modabber A, Gerressen M. 2016, J Oral Maxillofac Surg , Vol. 74, pp. 2230-2238.
6. Surgical Approaches to the Infraorbital Rim and Orbital Floor: The Case for the subtasal approach. Wilson S, Ellis E $3^{\text {rd }}$. 1, Jan 2006, J Oral Maxillofac Surg., Vol. 64, pp. 104-107.

7. Toward CT-based fracture treatment. Manson PN, Markowitz B, Mirvis S, et al:. 1990, Plast Reconstr Surg, Vol. 85, pp. 202-207.

8. AnalysisofTreatmentforIsolatedzygomaticomaxillary complex fractures. P, Manson. 1996 : s.n., J Oral Maxillofac Surg, Vol. 54, pp. 400-401.

9. Analysis of treatment for isolated zygomaticomaxillary. Ellis E, KittidumkerngW. 1996, J Oral Maxillofac Surg, Vol. 54, pp. 386-391.

10. 3D assessment of morbidity associated with lower eyelid incisions in orbital trauma. Y. H. Nunu, A. Bell, S. McHugh,K. F. Moos, A. F. Ayoub. 2007, Int J. Oral Maxillofac. Surg., Vol. 36, pp. 680-686.

11. Treatment of 813 zygoma-lateral orbital complex fractures. New aspects. Zingg M, Chowdhury K, Lädrach K, Vuillemin T, Sutter F, Raveh J. 6, Jun 1991, Arch Otolaryngol Head Neck Surg. , Vol. 117, pp. 611-620.

12. Association Between Ocular Injuries and Internal Orbital Fractures. He D, Blomquist PH, Ellis E . 2007, J Oral Maxillofac Surg, Vol. 65, pp. :713-720.

13. An analysis of 2,067 cases of zygomatico-orbital fracture. Ellis E, El-Attar A, Moos K. 1985, J Oral Maxillofac Surg, Vol. 43, pp. 417- 420.

14. Status of the Internal Orbit Afte rReduction of Zygomaticomaxillary Complex Fractures. 2004, J Oral Maxillofac Surg, Vol. 62, pp. 275-283.

15. The Effects of Zygomatic Complex Fracture on Masseteric Muscle Force. Dal Santo F, Ellis E $3^{\text {rd }}$, Throckmorton GS. 1992, J Oral Maxillofac Surg, Vol. 50, pp. 791-799. 